\title{
Structure-Specific and Individual-Dependent Metabolization of Human Milk Oligosaccharides in Infants: A Longitudinal Birth Cohort Study
}

Fangjie Gu, Shuang Wang, Roseriet Beijers, Carolina de Weerth, and Henk A. Schols*

Cite This: J. Agric. Food Chem. 2021, 69, 6186-6199

Read Online

ACCESS | Lill Metrics \& More | 回 Article Recommendations ｜ＳＳ Supporting Information

ABSTRACT: To follow human milk oligosaccharide (HMO) biosynthesis and in vivometabolization, mother milk and infant feces from 68 mother-infant dyads at 2, 6, and 12 weeks postpartum were analyzed, with 18 major HMOs quantitated. Fucosylated and neutral core HMO levels in milk were dependent on mothers' Lewis/Secretor status, whereas most sialylated HMO levels were independent. Infant fecal excretion of HMOs gradually declined with age, especially for neutral core structures. Although decreasing in absolute concentrations in milk during lactation, the relative abundance of total fucosylated HMOs increased in both milk and feces. Mono-fucosylated HMOs were more consumed than those decorated with two fucose moieties. More ( $\alpha 2-3)$-sialylated HMOs were degraded than ( $\alpha 2-6)$-sialylated HMOs. The transition speed of HMO metabolization from nonspecific or structurespecific consumption stage to the complete consumption stage was individual-dependent. Variation was associated with mode and place of delivery, where caesarean section or early exposure to hospital environment delayed the transition.

KEYWORDS: consumption pattern, delivery mode, Lewis, Secretor, prebiotics

\section{INTRODUCTION}

Human milk oligosaccharides (HMOs) have gained increasing research interest due to their high abundance in human milk $(5-15 \mathrm{~g} / \mathrm{L}),{ }^{1}$ as well as the health benefits for infants' immune system, ${ }^{2}$ gut microbiota establishment, ${ }^{3}$ and cognitive development. ${ }^{4,5}$ After ingestion by infants, HMOs are minimally digested in the upper gastrointestinal (GI) tract $(<5 \%),{ }^{6,7}$ or absorbed through the brush border into the circulation system and excreted into urine $(0.5-2 \%){ }^{8,9}$ Instead, HMOs are mainly utilized by intestinal bacteria as fermentation substrate, with the remaining HMOs, if any, excreted into the feces. ${ }^{10,11}$ Colonization of the infant's intestine by microorganisms initiates at delivery or already in utero ${ }^{12,13}$ and follows a stage-specific development. ${ }^{14}$ Bifidobacterium species are known to be predominant in breast-fed infants' colon, partially attributed to their HMO assimilation abilities. ${ }^{15,16}$ Despite interindividual differences, gut microbiota composition progresses toward adult-like patterns in the first 3 years of life with microbial richness and diversity being highly increased. ${ }^{14} \mathrm{~A}$ healthy gut microbiota in early life is vital for maintaining normal functions of both metabolic and immune systems, both having a persisting long-term influence. ${ }^{17}$ Several factors have been reported as driving force to shape the gut microbiota of infants, and these include mode of delivery, antibiotic exposure, premature birth, and feeding method. ${ }^{18,19}$ Infants who are exclusively breast-fed, formula-fed, or mixed-fed showed different transitions across developmental stages. ${ }^{13}$ HMOs are a group of complex glycans comprising over 240 reported structures, all built from the five monosaccharides glucose, galactose, $\mathrm{N}$-acetylglucosamine, fucose (Fuc), and $\mathrm{N}$ acetylneuraminic acid (Neu5Ac). ${ }^{20-23}$ The core structures of HMOs start with lactose, extended at the nonreducing end with one or more specific disaccharides, i.e., lacto- $N$-biose or $\mathrm{N}$-acetyllactosamine, in a linear or a branched manner. ${ }^{1}$ Fucosylated HMOs or sialylated HMOs are formed when fucose (Fuc) or $\mathrm{N}$-acetylneuraminic acid (Neu5Ac; also referred to as sialic acid) residues are covalently bound to the core structures, respectively. HMOs can be further grouped depending on the number and linkage types of these fucose/ sialic acid moieties, e.g., Fuc-( $\alpha 1-2)$ - or ( $\alpha 1-3)$ - or ( $\alpha 1-4)$ substituted HMO groups, mono- vs di-fucosylated HMOs, or $\mathrm{Neu} 5 \mathrm{Ac}(\alpha 2-3)$ vs ( $\alpha 2-6)$-substituted HMOs. Composition and amounts of HMOs secreted into the milk by women are influenced by maternal genetics and lactation duration. Considerable amounts of Fuc- $(\alpha 1-2)$ and Fuc- $(\alpha 1-3 / 4)$ structures are secreted by mothers with an active Secretor $(\mathrm{Se})-$ and Lewis (Le)- gene, respectively. ${ }^{24}$ When mothers are non-Secretors, or Lewis-negative, Fuc- $(\alpha 1-2)$ and Fuc- $(\alpha 1$ $3 / 4)$ structures are nearly absent in their milks, respectively. HMOs with different structural elements showed distinct temporal changes regarding concentrations in human milk with lactation duration. ${ }^{25}$

Previous studies showed that specific HMO structures undergo different metabolic fates in the GI tract. ${ }^{26,27}$ Coincident with the temporal development of infant gut microbiota, metabolization of HMOs follows a three-stage

Received: November 27, 2020

Revised: April 9, 2021

Accepted: May 6, 2021

Published: May 25, 2021

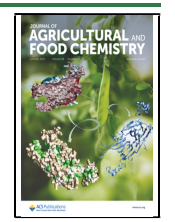


pattern, as proposed by Albrecht et al. based on fecal HMO profiles at several time points. ${ }^{28}$ Infant feces in the first weeks postpartum still contained either neutral HMOs or sialylated ones. This was followed by a transition to HMO-derived metabolites, and finally to the absence of any HMO-related structure with the introduction of solid foods over 6 months of age. $^{28}$ However, contradictory findings or exceptional cases have been reported. Where Chaturvedi et al. found that HMO patterns in infant feces highly resembled that in mother milk, ${ }^{29}$ Dotz et al. reported on an infant showing higher abundances of fecal neutral HMOs at 7 months postpartum compared to that at 2 months. ${ }^{30}$ Furthermore, we recognized three distinct HMO consumption patterns already at 1 month postpartum in another birth cohort that were (1) complete consumption, (2) considerable consumption on specific structures, and (3) low consumption. ${ }^{27}$ However, these studies have in common that they were limited by either small sample sizes, single time points, or did not analytically differentiate between structural isomers.

To further investigate HMO metabolization by infant gut microbiota in a longitudinal manner, human milk and paired infant fecal samples from a longitudinal birth cohort study collected at 2, 6 , and 12 weeks postpartum were analyzed, with 18 major HMOs quantitated. Temporal changes of $\mathrm{HMO}$ profiles in human milk were examined, as well as metabolization of different structural HMO groups during the first 12 weeks of life. Progresses of HMO consumption patterns were compared among infants, to find out possible factors that influence these different transitional stages.

\section{MATERIALS AND METHODS}

Birth Cohort and Sample Collection. The study design of the BINGO study has been published before, ${ }^{31,32}$ where BINGO stands (in Dutch) for Biological Influences on Baby's Health and Development. The study recruited participants in the NijmegenArnhem region in the Netherlands with the aim to identify prenatal and early postnatal factors that predict infant health and development. The ethical committee of the Faculty of Social Sciences of the Radboud University approved the study [ECSW2014-1003-189], and informed consent was signed by participants. In general, healthy mothers and infants who were delivered $>37$ weeks of gestational age were eligible participants, with more detailed initial and postnatal inclusion criteria described elsewhere. ${ }^{31,32}$ The infants included in the current study were born between December 2014 and November 2016. Human milk and infant fecal samples were collected when each infant reached 2, 6, and 12 weeks of age. Collection of milk samples from the breast into sterile cups was performed by mothers with hand expression before the first morning feeding. Infant feces were collected within $48 \mathrm{~h}$ after milk collection, being transited from diapers into sterile stool vials. These samples were temporarily stored in the participants' home freezers until the samples from all three time points had been collected. At the end of this collection period (i.e., after 12 weeks postpartum), the samples were transferred to a $-80^{\circ} \mathrm{C}$ laboratory freezer until further HMO analysis. More details about sample collection procedures can be found in a previous article. ${ }^{33}$ In total, 186 human milk samples and 187 infant fecal samples were received from 75 mother-infant dyads. Thirteen fecal samples corresponding to five mother-infant pairs were excluded from further analysis because the infants were formula-fed at all three time points, without any breast milk samples collected. Six milk samples and four fecal samples from two pairs were excluded because the gestational age at birth was not provided. Eighteen fecal samples from nine infants were excluded due to either unknown feeding type or mislabeling. The remaining 180 milk samples and 152 fecal samples were analyzed for HMO concentrations.
HMO Quantitation. HMO concentrations of the milk and fecal samples were quantitated based on the method as described by Gu et al. with some adaptations. ${ }^{27}$ Briefly, milk samples were diluted twofold, followed by centrifugation at $4{ }^{\circ} \mathrm{C}(21000 \mathrm{~g}, 20 \mathrm{~min})$ to remove the fat layer. Subsequently, a solid-phase extraction (SPE) procedure was applied to extract oligosaccharides, using Supelclean ENVI-Carb $250 \mathrm{mg} / 3 \mathrm{~mL}$ cartridges (Sigma-Aldrich; St. Louis, MO). Two fractions were lyophilized and rehydrated for further analysis: the fraction containing 3-fucosyllactose (3FL) was analyzed by highperformance anion exchange chromatography-pulsed amperometric detection (HPAEC-PAD), whereas the other one containing all other HMOs was analyzed by porous graphitized carbon-liquid chromatography-mass spectrometry (PGC-LC-MS). Reduction of oligosaccharides with sodium borohydride overnight, and a following purification step with SPE was performed before loading samples to PGC-LC-MS. Thawed fecal samples were weighed and diluted to $100 \mathrm{mg} / \mathrm{mL}$ with Milli-Q water, followed by centrifugation. Then, an aliquot of $100 \mu \mathrm{L}$ supernatant was taken directly to reduction and purification steps, and was analyzed by PGC-LC-MS.

An ICS 5000 system (Dionex, Sunnyvale, CA) equipped with a CarboPac PA-1 column $(250 \mathrm{~mm} \times 2 \mathrm{~mm}$ ID $)$ and a CarboPac PA guard column $(25 \mathrm{~mm} \times 2 \mathrm{~mm} \mathrm{ID})$ was employed for $3 \mathrm{FL}$ analysis. The column temperature was maintained at $20{ }^{\circ} \mathrm{C}$, and the flow rate was set as $0.3 \mathrm{~mL} / \mathrm{min}$. The injection volume was $10 \mu \mathrm{L}$. Mobile phase A was $0.1 \mathrm{M} \mathrm{NaOH}$, and mobile phase $\mathrm{B}$ was $1 \mathrm{M} \mathrm{NaOAc}$ in $0.1 \mathrm{M} \mathrm{NaOH}$. The elution profile was as follows: $0-10 \mathrm{~min}, 0-10 \%$ B; $10-10.1 \mathrm{~min}, 10-100 \% \mathrm{~B}$; $10.1-15 \mathrm{~min}, 100 \% \mathrm{~B}$; $15-15.1 \mathrm{~min}$, $100-0 \% \mathrm{~B} ; 15.1-30 \mathrm{~min}, 0 \% \mathrm{~B}$. The eluted oligosaccharides were monitored by a pulsed amperometric detector (Dionex).

An Accela ultrahigh-pressure liquid chromatography system (Thermo Scientific, Waltham, MA) equipped with a Thermo Hypercarb column $\left(100 \times 2.1 \mathrm{~mm}^{2}, 3 \mu \mathrm{m}\right.$ particle size $)$ preceded by a Hypercarb guard column $\left(10 \times 2.1 \mathrm{~mm}^{2}, 3 \mu \mathrm{m}\right.$ particle size $)$ was used to analyze HMOs present in the reduced samples. Eluent A was $1 \%(\mathrm{v} / \mathrm{v})$ acetonitrile $(\mathrm{ACN})$ in water containing $0.1 \%(\mathrm{v} / \mathrm{v})$ formic acid; eluent B was ACN containing $0.1 \%(\mathrm{v} / \mathrm{v})$ formic acid. The elution profile was as follows: $0-5 \mathrm{~min}, 3 \% \mathrm{~B} ; 5-22 \mathrm{~min}, 3-20 \% \mathrm{~B}$; $22-32 \mathrm{~min}, 20-40 \% \mathrm{~B}$; followed by washing with $100 \%$ B for $10 \mathrm{~min}$ and equilibrating with $3 \% \mathrm{~B}$ for $21 \mathrm{~min}$. Temperatures of the column oven and sample tray were set at 25 or $10{ }^{\circ} \mathrm{C}$, respectively. The injection volume was $5 \mu \mathrm{L}$. The flow rate of the washing step and the first $10 \mathrm{~min}$ of equilibration was $300 \mu \mathrm{L} / \mathrm{min}$, and that of the rest of elution was $200 \mu \mathrm{L} / \mathrm{min}$. The Velos Pro mass spectrometer (Thermo Scientific) with an electrospray ionization probe was operated in negative-ion mode over a mass-to-charge ratio $(\mathrm{m} / \mathrm{z})$ range of 300$2000 \mathrm{Da}$. Chromeleon 7.1 (Dionex) and XCalibur 4.3 (Thermo Scientific) were used for processing data obtained from HPAEC-PAD or LC-MS, respectively.

A number of HMOs was identified by comparing retention times and mass-to-charge ratios $(\mathrm{m} / \mathrm{z})$ to commercial standards, including 3-FL, $2^{\prime}$-fucosyllactose ( $\left.2^{\prime} \mathrm{FL}\right)$, lacto- $N$-fucopentaose I (LNFP I), lacto- $N$-fucopentaose II (LNFP II), lacto- $N$-fucopentaose III (LNFP III), lacto- $N$-fucopentaose V (LNFP V), lacto- $N$-difucohexaose I (LNDFH I), difucosyllactose (DFL), lacto- $N$-tetraose (LNT), lacto$N$-neotetraose (LNnT), lacto- $N$-hexaose (LNH), lacto- $N$-neohexaose $(\mathrm{LNnH}), 3^{\prime}$-sialyllactose $\left(3^{\prime} \mathrm{SL}\right), 6^{\prime}$-sialyllactose $\left(6^{\prime} \mathrm{SL}\right)$, sialyl-lacto- $N$ tetraose a (LST a), sialyl-lacto- $N$-tetraose b (LST b), and sialyl-lacto$\mathrm{N}$-tetraose c (LST c). Calibration curves were constructed for each HMO for quantitation, based on integrated peak area from PAD or MS signals. Two structural isomers, LNT and LNnT, were quantitated together because of co-elution. Beyond the abovementioned HMOs, an adjacent peak following LNDFH I with the same $m / z$ value was identified as lacto- $N$-difucohexaose II (LNDFH II), consistent with the literature. ${ }^{22}$ Quantitation of LNDFH II was based on the calibration curve of LNDFH I, by assuming a similar response factor.

Data and Statistical Analysis. The presence or (near) absence of $2^{\prime} \mathrm{FL}$ and LNFP I in human milk was used as indicators of a mother's Secretor status, whereas LNFP II acted as an indicator of Lewis blood groups. ${ }^{27}$ The mothers were assigned to four milk groups 
based on their milk oligosaccharide profiles: milk group 1, Lewispositive Secretor $(\mathrm{Le}+\mathrm{Se}+)$, milk group 2, Lewis-positive non-Secretor ( $\mathrm{Le}+\mathrm{Se}-)$; milk group 3, Lewis-negative Secretor (Le-Se+); milk group 4, Lewis-negative non-Secretor (Le-Se-). In the current study, the total HMO was defined as the sum of the 18 annotated HMOs. The sum of 2'FL, LNFP I/II/III/V, DFL, LNDFH I/II, and 3FL was defined as total fucosylated HMOs; the sum of LNT, LNnT, LNH, and $\mathrm{LNnH}$ was defined as total neutral core HMOs; the sum of $3^{\prime} \mathrm{SL}$, $6^{\prime} \mathrm{SL}$, and LST $\mathrm{a} / \mathrm{b} / \mathrm{c}$ was defined as total sialylated HMOs. Furthermore, the group of Fuc- $(\alpha 1-2)$ structural HMOs included $2^{\prime}$ FL, LNFP I, DFL, and LNDFH I; Fuc- $(\alpha 1-3 / 4)$ group included 3FL, LNFP II/III/V, and LNDFH II; mono-Fuc included $2^{\prime} \mathrm{FL}$, LNFP I/II/III/V, and 3FL; di-Fuc included DFL, and LNDFH I/II. The sialylated Neu5Ac- $(\alpha 2-3)$ HMOs contained $3^{\prime}$ SL and LST a, whereas the NeuSAc- $(\alpha 2-6)$ group included 6 'SL and LST b/c. Relative abundances of total fucosylated, neutral core, and total sialylated HMOs were calculated as the concentrations of each group divided by the sum of all individual HMO concentrations. Relative abundances of Fuc- $(\alpha 1-2)$, Fuc- $(\alpha 1-3 / 4)$, mono-Fuc, and di-Fuc structures were calculated as the concentration of each group divided by the total of fucosylated HMOs. Relative abundances of Neu5Ac$(\alpha 2-3)$ and Neu5Ac- $(\alpha 2-6)$ groups were calculated as the concentration of each group divided by the total of sialylated HMOs.

To study longitudinal changes of HMO profiles in human milk, all milk samples were included for statistical analysis $(n=180)$. With respect to longitudinal fecal HMO profiles, only fecal samples collected from the infants who were exclusively breast-fed at that specific time point were included $(n=115)$. To compare the relative abundances of different structural HMO groups between human milk and infant fecal samples, mother-infant pairs were excluded if total fecal HMO concentrations were less than $1 \mu \mathrm{g} / \mathrm{mg}$, considering full utilization. Continuous variables, including age postpartum, gestational age, birthweight, HMO absolute concentrations, as well as relative abundances in milk and feces, were compared between milk group 1 and milk group 2 with Mann-Whitney tests, or among the three time points within each milk group with Kruskal-Wallis with post hoc stepwise multiple comparisons. Pearson's $\chi^{2}$ test was used to compare categorical variables, i.e., gender, delivery mode, and delivery place, between the milk groups. The calculation of averages, standard deviations (SD), percentages, and the above-mentioned comparisons was performed with SPSS Statistics version 26 (IBM Corp., Armonk, NY). The Spearman rank-order correlation coefficients with associated $p$-values between individual HMOs in human milk samples were calculated using scipy.stats.spearmanr, then visualized with heatmap using matplotlib.pyplolt in Python (version 3.7.7). Hierarchical cluster analysis (HCA) was performed for milk groups 1 and 2 separately, based on relative compositional changes of each quantitated HMO between milk and paired fecal samples at all of the three time points, with only mother-infant pairs that had full sets of samples included. Initial relative compositional changes were calculated by subtracting the relative abundance of individual HMO in milk from the corresponding relative abundance in paired feces and then dividing by the former value. All of the positive values were normalized to $0-100 \%$, by defining the initial maximum positive values in each sample as $100 \%$. When the total HMO concentration in a fecal sample was below $1 \mu \mathrm{g} / \mathrm{mg}$, it was considered as complete degradation, and the relative composition changes of all individual HMO structures were designated as $-100 \%$. The relative compositional changes were visualized as heatmaps, using color scales function in Microsoft Excel 2019. The influence of maternal-infant variables on relative HMO compositional changes at each time point and overall were evaluated by multiple response permutation procedures (MRPP). HCA and MRPP were conducted in R (version 3.4.0), using factoextra and vegan packages, respectively. Results were considered as statistically significant when $p$-values were less than 0.05 , or highly significant when less than 0.001 .

\section{RESULTS}

Maternal and Infant Characteristics. Among the 68 pairs, human milk samples from 51 mothers $(75 \%)$ contained considerable concentrations of $2^{\prime} \mathrm{FL}$, LNFP I, and LNFP II, indicating active Lewis (Le)- and Secretor $(\mathrm{Se})-$ genes, therefore assigned to milk group $1(\mathrm{Le}+\mathrm{Se}+)$. The peaks of $2^{\prime} \mathrm{FL}$ and LNFP I in milks of the remaining 17 pairs (25\%) were all found to be (nearly) absent, while LNFP II was present in considerable levels, based on which these pairs were assigned to milk group $2(\mathrm{Le}+\mathrm{Se}-)$. Mothers with genotype Se $+\mathrm{Le}-$ or $\mathrm{Se}-\mathrm{Le}-$ were absent among the pairs. Maternal and infant characteristics are summarized and compared in Table 1.

Table 1. Characteristics of Study Subjects from Milk Group $1(\mathrm{Le}+\mathrm{Se}+)$ and Milk Group $2(\mathrm{Le}+\mathrm{Se}-)^{a}$

\begin{tabular}{|c|c|c|c|}
\hline variables & $\begin{array}{c}\text { milk group } 1 \\
(n=51)\end{array}$ & $\begin{array}{l}\text { milk group } 2 \\
(n=17)\end{array}$ & $p$-value \\
\hline \multicolumn{4}{|l|}{$\begin{array}{l}\text { age postpartum, day, mean } \\
\text { (SD) }\end{array}$} \\
\hline week 2 & $15(1)^{b}$ & $15(2)^{d}$ & 0.169 \\
\hline week 6 & $43(1)^{b}$ & $43(2)^{c}$ & 0.557 \\
\hline week 12 & $85(5)^{b}$ & $85(3)^{b}$ & 0.716 \\
\hline $\begin{array}{l}\text { gestational age, week, mean } \\
\text { (SD) }\end{array}$ & $40.1(1)$ & $40.0(1)^{c}$ & 0.542 \\
\hline birthweight, $g$, mean (SD) & $3591(400)$ & $3580(456)^{c}$ & 0.652 \\
\hline gender, male, $n(\%)$ & $26(53)^{c}$ & $10(59)$ & 0.651 \\
\hline $\begin{array}{l}\text { delivery mode, vaginal, } n \\
(\%)\end{array}$ & $46(90)$ & $15(88)$ & 0.818 \\
\hline $\begin{array}{l}\text { delivery place, home, } n \\
(\%)^{e}\end{array}$ & $17(33)$ & $1(6)^{b}$ & 0.024 \\
\hline
\end{tabular}

${ }^{a}$ Data are presented as mean (standard deviation, SD), or number of subjects (percentages). ${ }^{b}$ One case with missing value. ${ }^{c}$ Two cases with missing values. ${ }^{d}$ Three cases with missing values. ${ }^{e}$ Other places, including hospital and clinic, are considered as hospital/clinic.

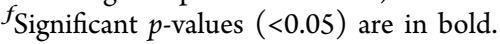

There are no significant differences between the two milk groups, regarding sample collection time points, gestational age at birth, birthweight, gender, and delivery mode. However, significantly fewer mothers from milk group 2 (6\%) delivered at home instead of clinic/hospital, compared to that from milk group $1(33 \%)$. The difference in delivery place should be taken into consideration when comparing HMO profiles between the two milk groups, as the exposure to hospital/ clinic surroundings could influence the initial acquisition of infant gut microbiota. ${ }^{12}$

Longitudinal Changes of HMO in Human Milk. A total of 18 major HMOs were quantitated in 180 human milk samples, the results of which are summarized in Table 2. Based on their averages, the most abundant HMOs in milk group 1 were $2^{\prime} \mathrm{FL}$ and $\mathrm{LN}(\mathrm{n}) \mathrm{T}$, followed by LNFP I and LNDFH I at week 2; the concentration of 3FL increased to be the second most abundant after $2^{\prime} \mathrm{FL}$ at week 12 , while the other abovementioned HMOs dropped in concentrations. In milk group 2 samples, $\mathrm{LN}(\mathrm{n}) \mathrm{T}, 3 \mathrm{FL}$, and LNFP II were the most predominant HMOs at all three time points, but HMOs containing ( $\alpha 1-2)$-linked fucose residues were hardly detected. All fucosylated HMOs, except for LNFP III at week 12, showed highly significant differences in concentrations between milk samples from group 1 and group 2 at all three time points. In general, ( $\alpha 1-2)$-linked fucosylated HMOs with or without other fucose residues were present in higher concentrations in milk group 1, whereas HMOs containing 


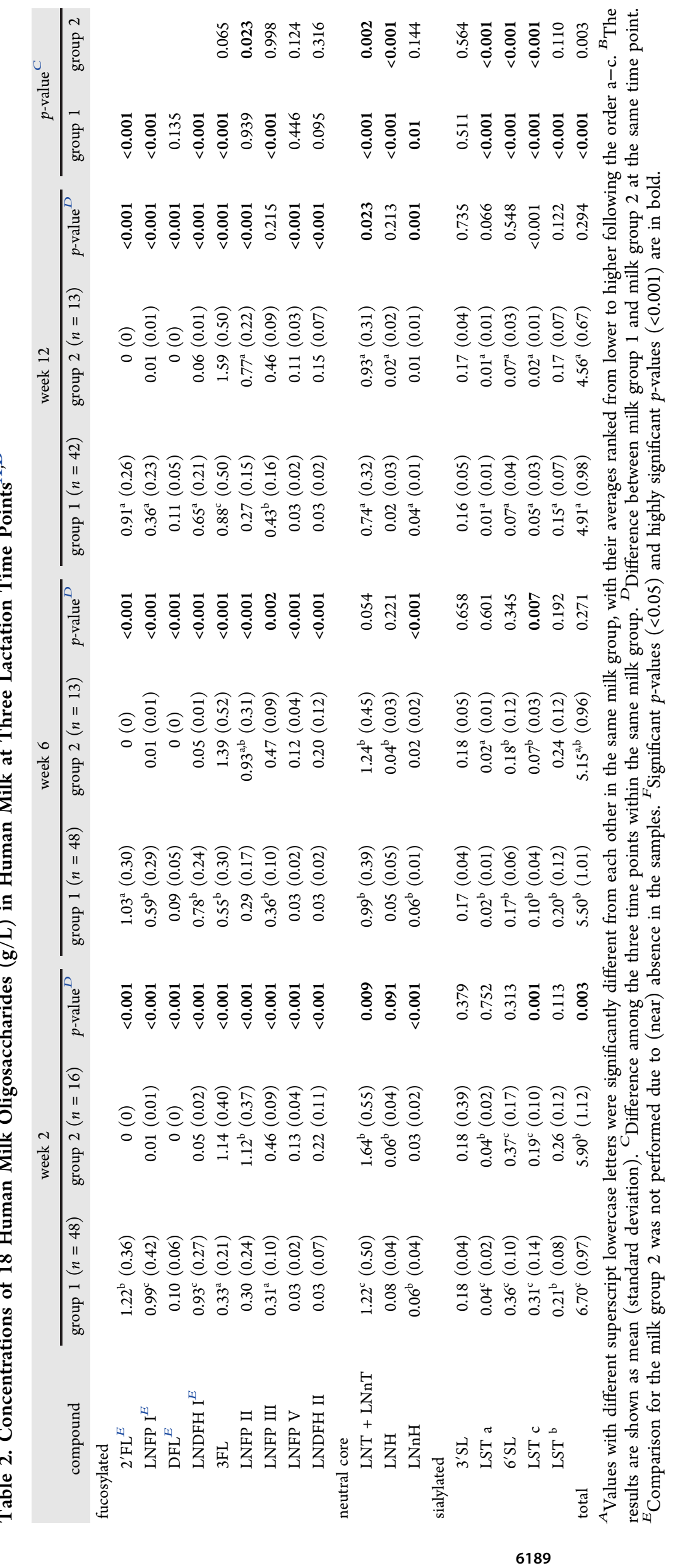




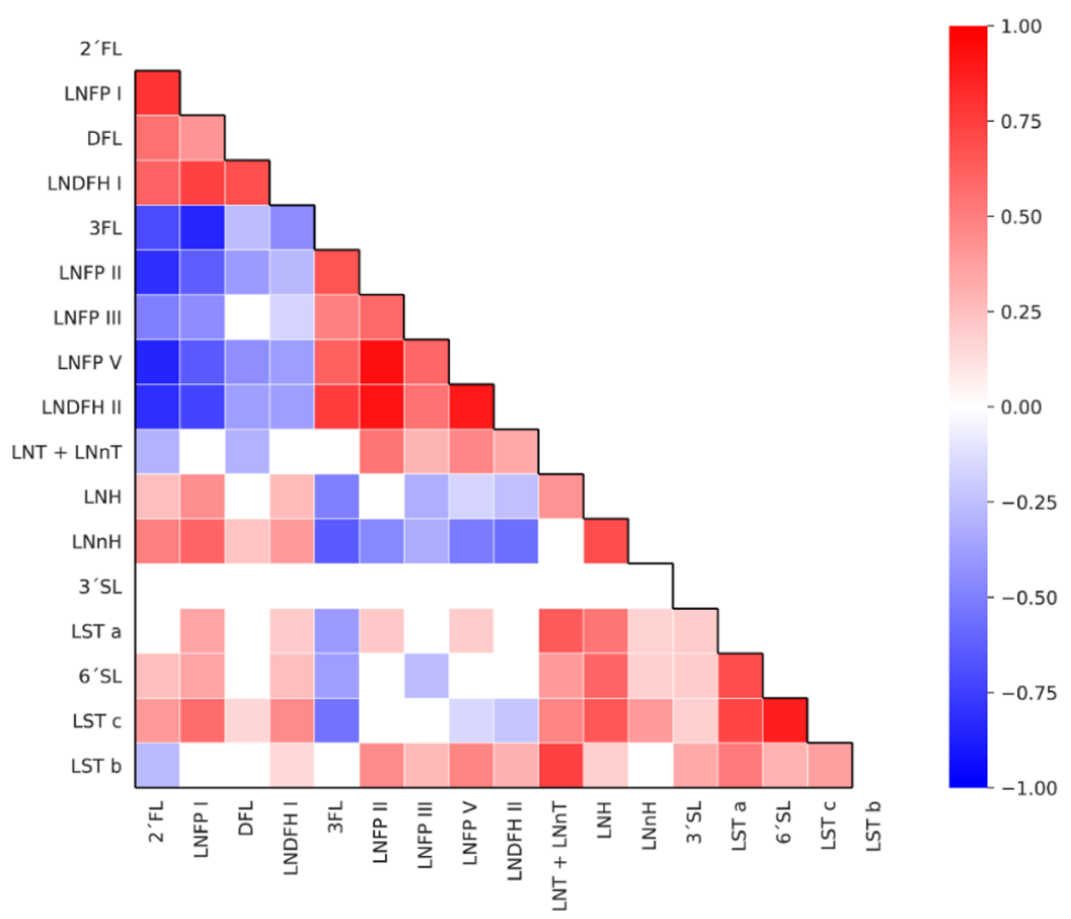

Figure 1. Spearman correlation heatmap between individual HMOs in milk during the first 12 weeks, including both milk groups at all time points. Color represents correlation from -1 (blue) to +1 (red) for cells with significant levels $(p<0.05)$, or white if not passing the significance threshold.

only ( $\alpha 1-3)$ - and/or ( $\alpha 1-4)$-linked fucose residues were higher in milk group 2 . As to the neutral core structures, milk group 1 contained less $\mathrm{LN}(\mathrm{n}) \mathrm{T}$ at weeks 2 and 12, but more $\mathrm{LNH}$ at week 2 and more $\mathrm{LNnH}$ along the first 12 weeks compared to milk group 2. No differences were found in sialylated HMOs between the two milk groups except for LST c, which was naturally more abundant in milk group 1 at all of the time points. Despite lacking ( $\alpha 1-2)$-linked structures, total HMO concentration secreted by milk group 2 mothers was found to be lower than milk group 1 only at week 2 (5.9 vs $6.7 \mathrm{~g} / \mathrm{L}$, respectively), while becoming comparable at later time points, though both groups showed decreases with longer lactation duration. This longitudinal reduction in concentrations was found for most individual HMOs, with a few exceptions: DFL remained stable over lactation in milk group 1; LNFP V, LNDFH II, and 3'SL were almost unchanged over time in both milk groups; 3FL levels increased over time in both milk groups yet the increase in milk group 2 was not significant $(p=$ $0.065)$; LNFP II reduced significantly in milk group 2 samples, but remained constant in group 1, while LNFP III showed exactly the opposite results. It should be noted that these observations over time were based on concentrations in human milk, instead of the total volume ingested by infants at that time, which would most likely lead to different conclusions on total intake, considering the increasing milk consumption when babies grew up. Borewicz et al. suggested a rather stable amount of HMO intake during the first 12 weeks postpartum, with an estimated changing daily intake of human milk over time. $^{33}$

To further investigate the relations between individual HMOs, correlations were calculated between each structure, involving all of the 180 milk samples, with significant results visualized as a heatmap in Figure 1. The heatmap clearly divided fucosylated HMOs into two groups, one group containing ( $\alpha 1-2)$-linked fucose (2'FL, LNFP I, DFL, and LNDFH I), the other devoid of ( $\alpha 1-2)$-linked fucose (3FL,
LNFP II/III/V, and LNDFH II). HMOs were positively correlated with each other within the same group $(r=0.42-$ $0.93)$, whereas negatively correlated with those from the other group $(r=-0.17$ to -0.86$)$. $\mathrm{LN}(\mathrm{n}) \mathrm{T}$ showed weak negative correlation with $(\alpha 1-2)$-fucosylated group $(r=-0.30)$ and positive correlation with ( $\alpha 1-3 / 4)$-fucosylated group $(r=$ 0.29-0.54); LNH and LNnH showed the opposite correlations with the two fucosylated groups $(r=0.23-0.61$, or -0.17 to -0.64 , respectively), although also belonging to the same neutral core group as $\mathrm{LN}(\mathrm{n}) \mathrm{T}$. These observations were consistent with their different concentrations detected in milk samples from milk group 1 and milk group 2. Hardly any strong correlations appeared for sialylated HMOs and fucosylated HMOs, except for LST c, which was positively correlated with LNFP I $(r=0.57)$ and negatively correlated with 3FL $(r=0.55)$, partly due to its higher abundance in milk group 2 milks. Except 3 'SL, which was found to be independent of all other HMOs, other sialylated structures were in general positively correlated with core HMOs and other sialylated HMOs, with the strongest correlations seen among $6^{\prime}$ SL, LST a, and LST c $(r=0.70-0.88)$, and between LST $\mathrm{b}$ and $\mathrm{LN}(\mathrm{n}) \mathrm{T}(r=0.74)$.

The 18 annotated HMOs were further grouped depending on their common structural elements, i.e., fucosylated group, neutral core group, sialylated group, ( $\alpha 1-2)$-fucosylated group, $(\alpha 1-3 / 4)$-fucosylated group, mono-fucosylated group, difucosylated group, ( $\alpha 2-3)$-sialylated group, and ( $\alpha 2-6)$ sialylated group, with the sum of each structural group compared between the two milk groups at three time points (Figure 2). Milk group 1 contained higher total fucosylated HMOs at weeks 2 and 12 than milk group 2, while milk group 2 contained higher total neutral core structures at week 2 than milk group 1, but comparable at other time points. Total concentrations of sialylated HMOs were not influenced by Secretor status, despite the higher abundance of LST c in milk group 1 (Table 2). Total core and total sialylated HMOs 


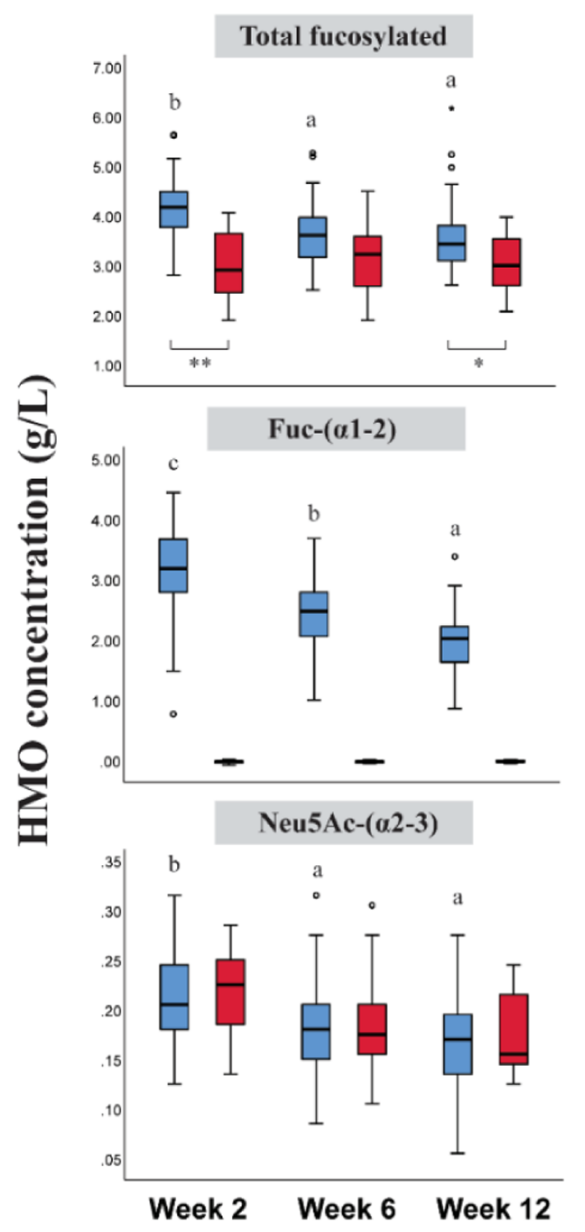

Milk group $1 \quad \square \quad$ Milk group 2
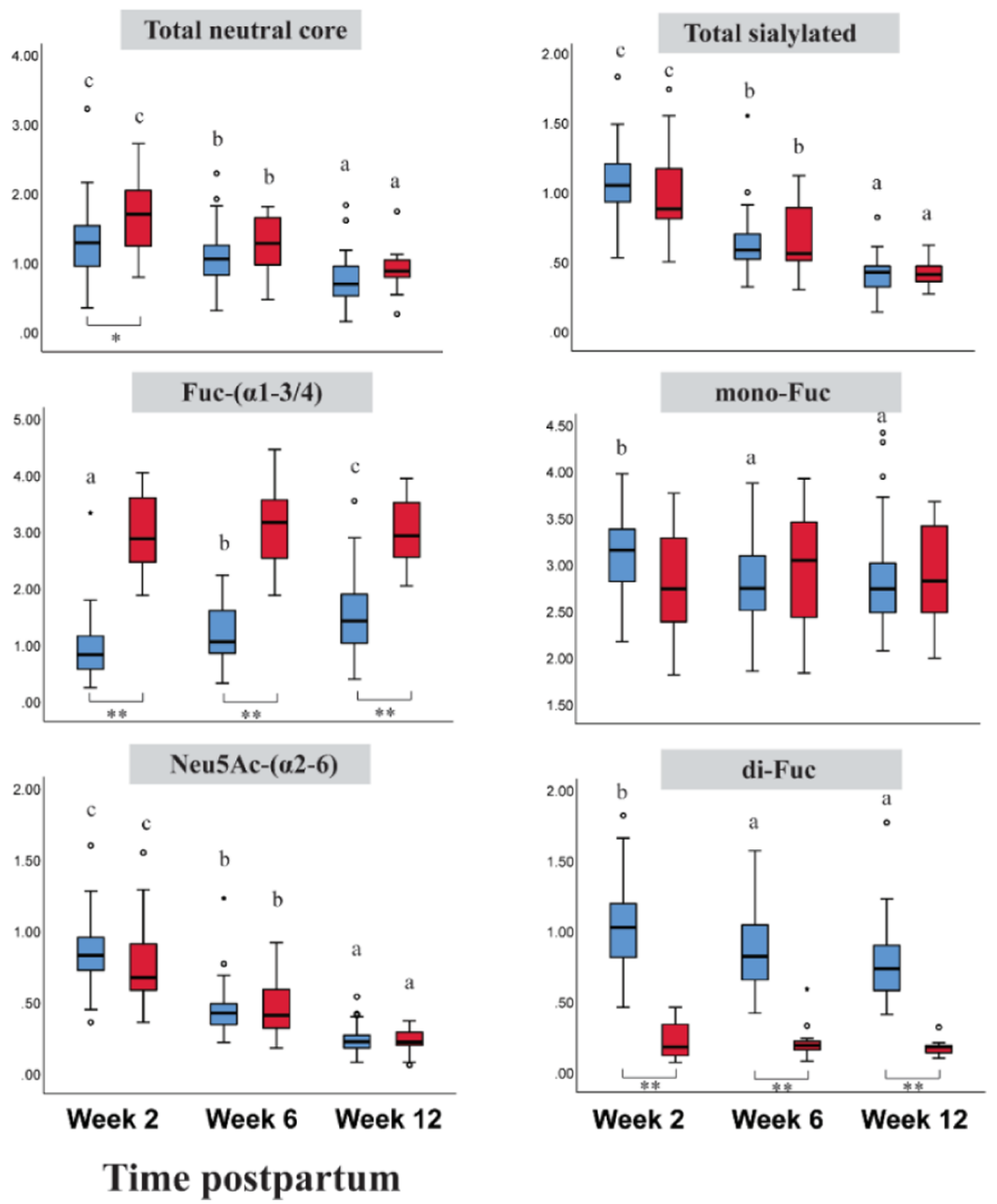

Figure 2. Concentrations of different HMO structural groups in milk samples at three time points from milk group 1 (Le+Se+, blue) or milk group 2 (Le+Se-, red). Box-whisker plots represent minimum, first quartile, median, third quartile, maximum, and outliers. Asterisks below the bars indicate significant differences $(* p<0.05, * * p 0.001)$ between the two milk groups at single time point. Different lowercase letters above the bars indicate significant differences $(p<0.05)$ from each other time point within the same milk group.

decreased with lactation time for both milk groups. Meanwhile, total fucosylated HMOs only reduced during weeks 2-6 for milk group 1 and remained unchanged from week 6 to 12 , or kept constant at all time points for milk group 2. Furthermore, the sums of ( $\alpha 1-3 / 4)$-fucosylated HMOs, mono-fucosylated HMOs, and di-fucosylated HMOs for milk group 2 all remained comparable over the course of lactation. Unlike milk group 2, ( $\alpha 1-2)$-fucosylated HMOs decreased while $(\alpha 1$ $3 / 4)$-fucosylated HMOs increased from week 2 to 12; monoand di-fucosylated HMOs both dropped from week 2 to 6 , but remained unchanged at the later time point. The two milk groups showed quite different distribution of fucosyl linkage types; nevertheless, no differences were found in their total mono-fucosylated HMO levels. As to di-fucosylated HMOs, a higher level was found in milk group 1 compared to the other milk group. Concentrations of ( $\alpha 2-3)$ - and ( $\alpha 2-6)$-sialylated HMOs were comparable between milk groups, indicating their independence from Secretor status of mothers. Albeit present in higher concentrations, ( $\alpha 2-6)$-sialylated HMOs decreased from week 2 to 12 in both milk groups, whereas ( $\alpha 2-3)$ sialylated HMO levels remained stable in milk group 2 and dropped only from week 2 to 6 in milk group 1 .
Longitudinal Changes of HMO in Infant Feces. HMO quantitation results of 115 infant fecal samples, which were collected at breast-fed time points, were included to investigate longitudinal changes of fecal HMO excretion, with results summarized in Table 3. It should be noted that concentration values given here were based on wet weight of feces, which were influenced by varied water contents of these feces. Only one infant (B119) was reported to have diarrhea problem at 6 weeks postpartum, while the rest of fecal samples were collected under healthy conditions. Among infants who were fed with Secretor milk (milk group 1), the most abundant HMOs in milk, $2^{\prime} \mathrm{FL}, \mathrm{LN}(\mathrm{n}) \mathrm{T}$, LNFP I, and LNDFH I, also accounted for the major part of HMOs present in their feces at week 2, with LNDFH I present in much higher concentration than the others. At later time points, fecal concentrations of 3FL, LNFP II/III, and LST b were relatively higher, whereas $\mathrm{LN}(\mathrm{n}) \mathrm{T}$ and LNFP I levels dropped most significantly; meanwhile, the LNDFH I level remained to be predominant. Infants who received non-Secretor milk (milk group 2) showed high concentrations of 3FL in their feces at all three time points, followed by LNFP II. These two HMOs were also most abundant in the corresponding milks. Another high-level 


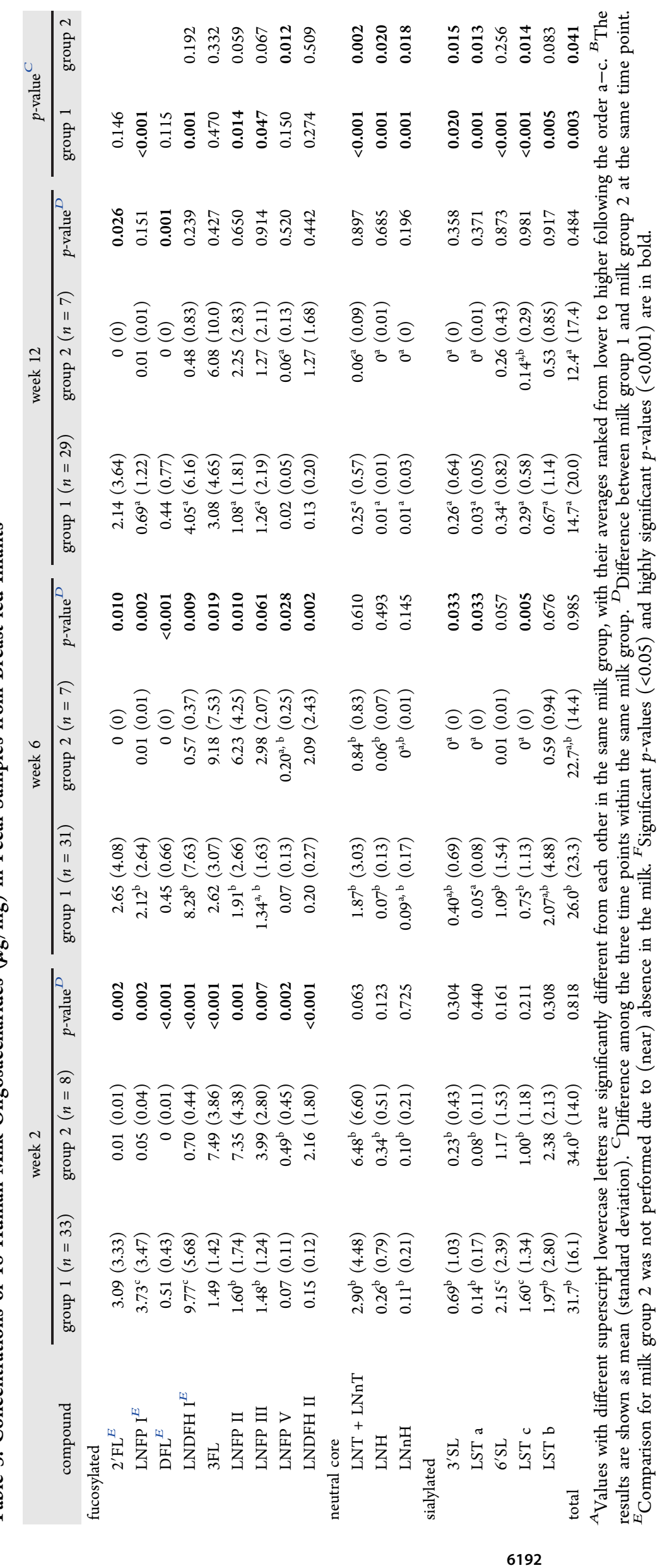



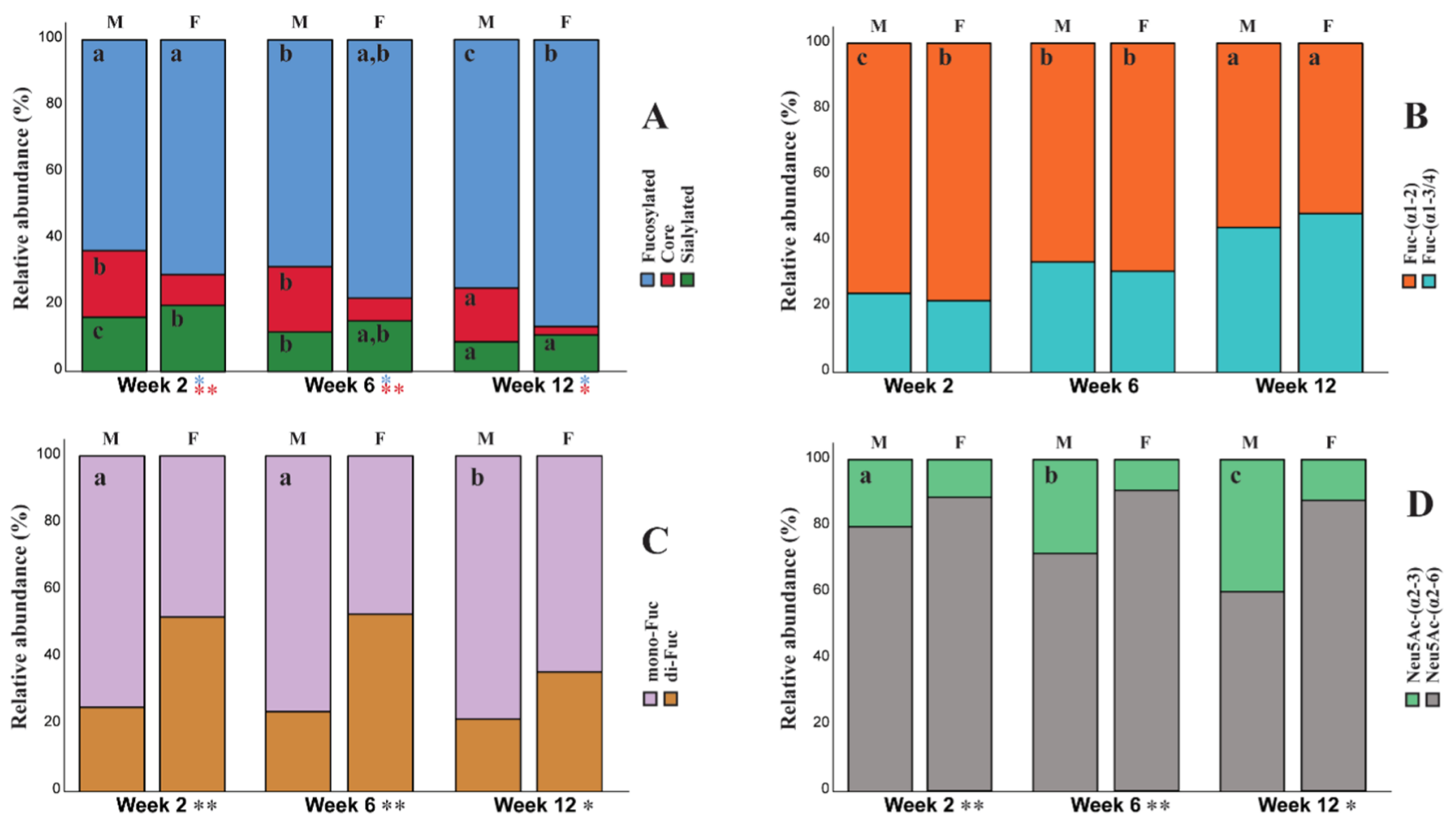

Figure 3. Relative abundances of different HMO structural groups ((A) total fucosylated, total core, and total sialylated HMO structures; (B) ( $\alpha 1$ 2)-fucosylated, and ( $\alpha 1-3)$-fucosylated HMO structures; (C) mono-fucosylated, and di-fucosylated HMO structures; (D) ( $\alpha 2-3)$-sialylated and ( $\alpha 2-6)$-sialylated HMO structures) in human milk (M) and in paired breast-fed infant feces (F), in which fecal HMOs were not completely utilized at week $2(n=30)$, week $6(n=26)$, and week $12(n=13)$. All subjects belong to milk group 1. Colored asterisks in (A) below the bars indicate significant differences $(* p<0.05, * *<0.001)$ for the structural groups of the same color, between milk and feces at single time point, while the black asterisks indicate significant differences for both structural groups in (B)-(D). Different lowercase letters in the bars indicate significant differences $(p<0.05)$ from each other time point within the same type of samples (milk, or feces), with each structural group annotated separately in (A), but both groups annotated by one letter in (B) $-(\mathrm{D})$.

HMO in the non-Secretor milk, $\operatorname{LN}(\mathrm{n}) \mathrm{T}$, was present as the third richest fecal HMO at week $2(6.48 \mu \mathrm{g} / \mathrm{mg})$; however, its average concentration strongly reduced to $0.84 \mu \mathrm{g} / \mathrm{mg}$ at week 6 , and to trace amounts $(0.06 \mu \mathrm{g} / \mathrm{mg})$ at week 12 . Four other HMOs, LNFP II, LNFP III, LNDFH II, and LST b, became the most concentrated ones at weeks 6 and 12. In general, infant fecal HMO concentrations displayed larger interindividual variations compared to that of human milk samples. A regular pattern was observed that included a gradual decrease in HMO concentrations excreted into the feces, especially of neutral core structures (LNT, LNnT, LNH, LNnH), despite their high concentrations in milk. The lower fecal HMO excretion reflects the development of infant gut microbiota with increased colonization of HMO-consuming bacteria. ${ }^{33}$ It is noted that the relative level of 3FL in milk group 1 feces increased slightly, although not significantly, from week 2 to 12. This even may indicate some microbial degradation of 3FL, since one should also consider the rising amount of milk 3FL ingested by the nursing infants.

Fecal concentrations of different HMO structural groups are shown in Figure S1. Despite high variations, total fucosylated HMOs were relatively more enriched and present in almost double the concentration of neutral core and sialylated HMOs. Furthermore, the longitudinal reduction in neutral core and sialylated HMOs was much more than that found for total fucosylated HMOs.

Longitudinal Changes of HMO Metabolization. To investigate metabolic fates of HMOs within the infant gut, we first compared the relative abundance of each structural group between human milk and infant feces at different time points (Figure 3). Inclusion criteria of data in Figure 3 were: milk group 1, exclusive breast-feeding, total HMO concentrations higher than $1 \mu \mathrm{g} / \mathrm{mg}$ in infant feces. Although the total level of fucosylated HMOs in milk decreased in absolute concentrations from week 2 to 6 , its relative abundance slightly increased over lactation (from 65 to $76 \%$ ). Both the absolute concentrations and relative abundances of the total neutral core HMOs (from 19 to 16\%) and total sialylated HMOs (from 16 to $9 \%$ ) in human milk decreased from week 2 to 12 . Similar trends in their relative abundances were observed in infant feces, where the total fucosylated structures accounted for the largest proportion at all three time points, and increased from $71 \%$ at week 2 to $86 \%$ at week 12 . Also, total sialylated HMOs and total neutral core HMOs dropped from 20 to $11 \%$, and from 9 to $3 \%$, respectively, over lactation. Although the core structures accounted for a higher proportion in milk than sialylated HMOs, their relative abundances were the opposite in feces. When comparing the distribution of the abovementioned three structural groups, fecal HMO profiles showed relatively more fucosylated structures, less neutral core, and comparable level of sialylated HMOs than milk at all of the time points. This difference implies a general utilization preference by infant gut microbiota, with neutral core HMOs being the most quickly consumed, followed by sialylated HMOs, and fucosylated HMOs being the least consumed during the first 12 weeks of life. Figure $3 \mathrm{~B}$ shows a rather comparable distribution for ( $\alpha 1-2)$ - and ( $\alpha 1-3 / 4)$-fucosylated HMOs between milk and feces for all time points, which 


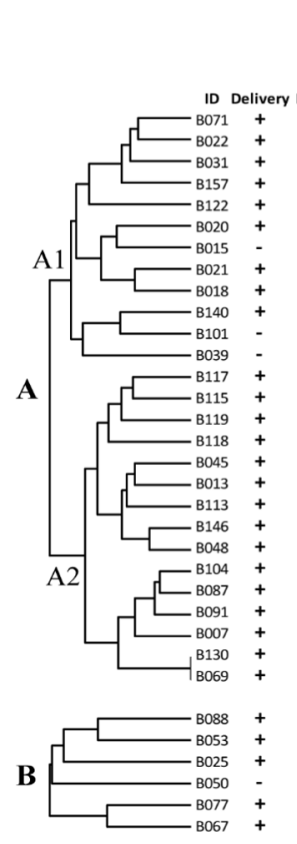

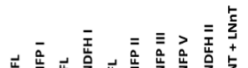

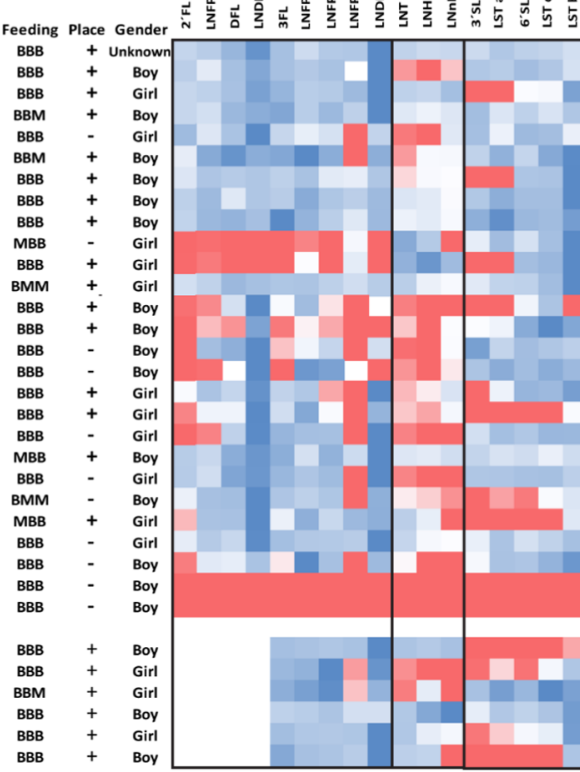

WEEK 2

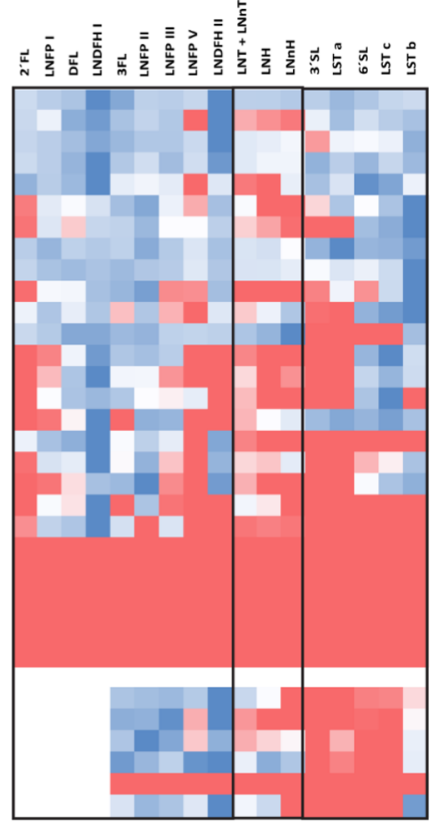

WEEK 6

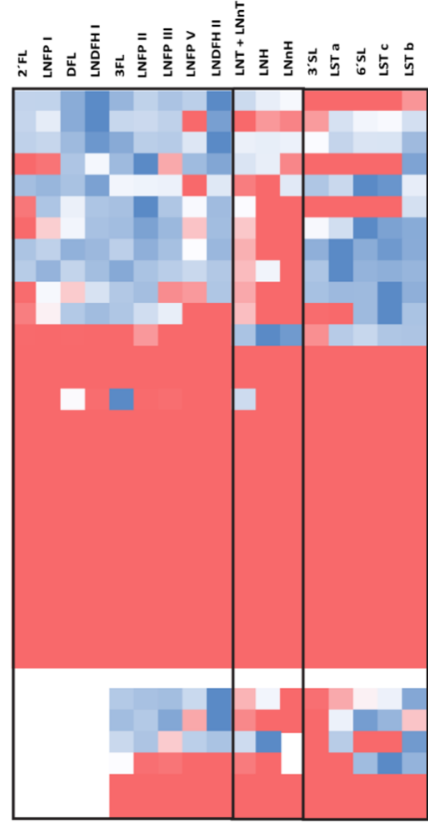

WEEK 12

Figure 4. Heatmap of relative HMO compositional changes between human milk samples and paired infant feces. The colors range from dark red to white to dark blue, representing $-100 \%$ (reduction) to $100 \%$ (increment) in feces compared to milk after normalization. Hierarchical clustering results of the mother-infant pairs are presented in a dendrogram for milk group 1 (A) and milk group 2 (B) separately. Delivery: +, vaginal delivery; - , caesarean section. Feeding: B/M, breast-feeding/mixed-feeding, with the three successive letters representing feeding type at weeks 2 , 6 , and 12 , respectively. Place: + , hospital/clinic; -, home.

indicates no preference in microbial consumption of these two structures in infants fed with Secretor milk. However, the proportion of ( $\alpha 1-2)$-fucosylated HMOs was much higher than that of ( $\alpha 1-3 / 4)$-fucosylated HMOs at week 2 ( $\sim 80$ vs $20 \%)$, although the abundance of these two groups became similar at week 12 . The comparison between $3-\mathrm{FL}$ and $2^{\prime} \mathrm{FL}$ in milk and fecal samples was made separately, and the results are shown in Figure S2. The utilization of $2^{\prime} \mathrm{FL}$ at week 2 seemed higher than that of 3-FL, but the differences were not large enough to allow general implications. The longitudinal distribution of mono-fucosylated and di-fucosylated HMOs remained relatively stable in both milk samples (75-79 vs $25-$ $21 \%)$ and fecal samples (48-65 vs $52-35 \%$ ), as can be seen in Figure 3C; however, the lower abundance of mono-fucosylated HMOs in feces compared to milk indicates that this structural group was more prone to microbial degradation than difucosylated structures. Within the sialylated HMOs (Figure 3D), ( $\alpha 2-3)$-sialylated structures accounted for only $21 \%$ of all sialylated HMOs in milk at week 2, while their proportion increased with prolonged lactation to $39 \%$ at week 12 . Interestingly, their distribution in fecal samples remained unchanged during all 12 weeks, with around 90\% being $(\alpha 2$ 6 )-sialylated group, which might be attributed to a growing assimilating ability of gut microbes toward ( $\alpha 2-3)$-sialylated HMOs with a longer duration of breast-feeding.

Besides the general trend in metabolization of different HMO groups, we also investigated the temporal HMO consumption pattern of individual infants, with results visualized in Figure 4. Only mother-infant pairs that provided milk and fecal samples from all of the three time points were included in the evaluation, with milk group 1 and milk group 2 clustered separately. Milk group 1 was divided over two main clusters (Figure 4A), with cluster A1 not reaching the complete
HMO consumption stage in the first 12 weeks of life, while cluster A2 showed complete consumption at week 12 the latest. Cluster A1 was further divided into three subclusters. Cluster A1-1 (B071, B022, B031, B157, and B122) showed growing consumption ability toward sialylated HMOs from week 2 to 12, while the fucosylated HMOs remained the most abundant in feces. Cluster A1-2 (B020, B015, B021, and B018) showed especially utilization of the neutral core structures, the degree of which enhanced with time; meanwhile, fucosylated and sialylated HMOs were predominantly excreted in feces. Subcluster A1-3 (B140 and B101) showed a preference for the consumption of fucosylated HMOs at week 2, but switched to utilization of neutral core structures at later time points. Conversely, B039 from the same subcluster A1-3 showed mainly consumption of sialylated HMOs at week 6 and changed to a fucosylated HMO consumption pattern at week 12. The other major cluster $\mathrm{A} 2$ also contained three subclusters. In general, subcluster A2-1 (B117, B115, B119, B118) showed utilization of neutral core structures, $2^{\prime} \mathrm{FL}$, LNFP I, 3FL, and LNFP V at week 2, with 3 'SL and LST a started to be consumed as well from week 6; finally, all HMOs were consumed at week 12. In contrast, subcluster A2-2 (B045, B013, B113, B146, B048) showed a stronger ability to degrade sialylated HMOs than fucosylated HMOs, especially those containing ( $\alpha 1-3 / 4)$-fucose moieties and at an early lactation stage. Both subclusters reached complete consumption stage later than 6 weeks postpartum. Two subjects from subcluster A2-3 (B104, B08, B091, B007, B130, B069) showed empty fecal HMO profiles already at 2 weeks postpartum, whereas the other four subjects from the same subcluster reached the final stage between weeks 2 and 6 . The relative HMO compositional changes of subjects from milk group 2 are shown in Figure 4B. Interindividual differences were also observed in 
this group. One subject from milk group 2 reached the complete consumption stage between week 2 and 6; B067 from milk group 2 could utilize all HMOs at week 12. All other samples from milk group 2 were not at the final stage of complete consumption at 12 weeks. All subjects from milk group 2 were delivered vaginally at hospital/clinic, except one (B50) that was delivered via caesarean section. There were still HMOs left in the B050 fecal sample at week 12, especially sialylated structures, $6^{\prime} \mathrm{SL}$, LST c, and LST b. Due to limited subject numbers, no clear differences were observed between mother-infant pairs from milk group 1 and milk group 2 .

When considering the compositional changes of individual HMOs, LNT, LNnT, LNH, and LNnH decreased the most at all of the three time points, followed by $2^{\prime} \mathrm{FL}$, LNFP I, LNFP $\mathrm{V}, 3^{\prime}$ SL, and LST a. The HMOs that showed the strongest increase in relative compositional levels were LST $b$ and LNDFH I and LNDFH II. This observation was consistent with our deduction on preferred degradation of different structural groups that neutral core, mono-fucosylated, and ( $\alpha 2$ 3)-sialylated HMOs were more prone to microbial consumption.

As discussed, quite diverse patterns of HMO metabolization among these subjects were observed, even within cluster A, where infants were all fed with Secretor milk. This indicates the influence of other determinants on HMO metabolization. It is noted that all of the caesarean-section-born infants belonged to the main cluster $\mathrm{A} 1$, and the infants in $\mathrm{A} 2$ were exclusively vaginally delivered. Furthermore, the occurrence of infants who were delivered at home was higher in cluster A2 compared to A1, whereas those delivered at hospital or clinic more likely clustered into A1. Therefore, multiple response permutation procedures (MRPP) were performed to identify any possible differences in HMO consumption clustering that were caused by maternal-infant variables (Table 4). Both delivery mode and delivery place had marginally significant influences on the overall consumption pattern across the 12 weeks. When considering single time points, the influence of delivery mode was marginally significant at week 2 and became significant at week 12. Likewise, delivery place only exerted significant influence starting from week 6 . Although both delivery mode and place had an effect on the initial acquisition of gut microbes, their influences on HMO-consuming abilities were more obvious with the babies' increasing age. No significant differences were found between boys and girls, or breast-fed and mixed-fed infants in the current subset of samples. Due to the limited samples size, we did not perform MRPP on milk group 2 .

\section{DISCUSSION}

Longitudinal Changes of HMO in Human Milk. The longitudinal setup of the Bingo birth cohort study enabled us to follow the changes in HMO profiles of human milk and infant fecal samples over the first 12 weeks of age in healthy Dutch mother-infant pairs. A total of 18 major HMOs were quantitated in the current study, despite the presence of over 100 structures. However, this is not regarded as a major problem, as it is generally accepted that more than $90 \%$ of total HMO concentrations in human milk was accounted for by 20 most abundant HMOs. ${ }^{34,35}$ In the current cohort, $75 \%$ of mothers were assigned to milk group $1(\mathrm{Le}+\mathrm{Se}+)$ and $25 \%$ to milk group 2 ( $\mathrm{Le}+\mathrm{Se}-$ ), which was comparable to the range of distribution as given in the literature $(70 \% \mathrm{Le}+\mathrm{Se}+$ vs $20 \% \mathrm{Le}$ $+\mathrm{Se}-){ }^{35-38}$ The averages of total HMO concentration were
Table 4. Multiple Response Permutation Procedures (MRPP) Results to Evaluate Any Differences in HMO Consumption Clustering of the Study Subjects, as Caused by the Listed Maternal-Infant Variables, at Each Time Point and Overall ${ }^{g}$

\begin{tabular}{|c|c|c|c|}
\hline time & variables & $A^{a}$ & $p$-value \\
\hline \multirow[t]{4}{*}{ week 2} & delivery mode ${ }^{b}$ & 0.01831 & 0.08 \\
\hline & delivery place ${ }^{c}$ & 0.01032 & 0.172 \\
\hline & infant gender ${ }^{d}$ & -0.01302 & 0.748 \\
\hline & feeding type $e^{e}$ & -0.01345 & 0.851 \\
\hline \multirow[t]{4}{*}{ week 6} & delivery mode & 0.01758 & 0.164 \\
\hline & delivery place & 0.05845 & 0.016 \\
\hline & infant gender & 0.00766 & 0.293 \\
\hline & feeding type & -0.01969 & 0.879 \\
\hline \multirow[t]{4}{*}{ week 12} & delivery mode & 0.04958 & 0.042 \\
\hline & delivery place & 0.05358 & 0.045 \\
\hline & infant gender & 0.01589 & 0.349 \\
\hline & feeding type & 0.00120 & 0.351 \\
\hline \multirow[t]{4}{*}{ overall } & delivery mode & 0.01992 & 0.074 \\
\hline & delivery place & 0.01904 & 0.076 \\
\hline & infant gender & -0.00107 & 0.497 \\
\hline & feeding pattern ${ }^{f}$ & -0.00080 & 0.465 \\
\hline
\end{tabular}

${ }^{a}$ Chance corrected within-group agreement $A$ values. ${ }^{b}$ Vaginal delivery vs caesarean section. ${ }^{c}$ Home/clinic vs home. ${ }^{d}$ Boy vs girl. ${ }^{e}$ Breast-feeding vs mixed-feeding. ${ }^{f_{B}}$ reast-feeding at all time points vs involvement of mixed-feeding at any time point. ${ }^{g}$ Only milk group 1 $(\mathrm{Le}+\mathrm{Se}+)$ samples were included for calculation. Significant $p$-values $(<0.05)$ are in bold, and marginally significant $p$-values $(<0.1)$ are italicized.

found to be 6.7 and $5.9 \mathrm{~g} / \mathrm{L}$ in milk from milk groups 1 and 2 at 2 weeks postpartum, respectively, both declined over lactation to 4.9 and $4.6 \mathrm{~g} / \mathrm{L}$ at 12 weeks. The concentrations were in the lower range of that given by widely cited reviews (5-15 g/L), ${ }^{1,39}$ although similar concentrations have been reported $\left(5-8 \mathrm{~g} / \mathrm{L}\right.$ in mature milk). ${ }^{40,41}$ Based on our results, the between-milk group differences were significant at early lactation stage and disappeared at week 6 . It should be noted that there were no Lewis-negative subjects included in our study, which might partly explain the slight difference with a previous finding, ${ }^{41}$ stating that the total HMO amount were still higher in Secretor's milk than non-Secretor at around 5 weeks postpartum.

Although one study pointed out that human milk samples were predominated by the non-fucosylated HMOs, ${ }^{10}$ our samples and another longitudinal study showed the clear abundancy of fucosylated HMOs in milk, ${ }^{42}$ with relative levels continuing to increase over lactation. The synthesis of fucosylated HMOs in the mammary gland required supply of both donor substrate nucleotide sugars and acceptor core structures, like $\mathrm{LN}(\mathrm{n}) \mathrm{T}, \mathrm{LN}(\mathrm{n}) \mathrm{H}$ as well as lactose. ${ }^{42}$ Due to competition for substrates, the (near) absence of ( $\alpha 1-2)$ fucosylated HMOs in milk group 2 was compensated by a higher amount of $\mathrm{LN}(\mathrm{n}) \mathrm{T}$ and ( $\alpha 1-3 / 4)$-fucosylated HMOs, which was also observed by other studies. ${ }^{42,43}$ However, mothers from milk group 1 seemed to produce a higher amount of $\mathrm{LNH}$ and $\mathrm{LNnH}$ in the milk compared to those from milk group 2, although both structures are only present in the milk at low concentrations. Both Azad et al. and Tonon et al. also reported higher levels of $\mathrm{LNH}$ and/or $\mathrm{LNnH}$ in $\mathrm{Le}+\mathrm{Se}$ + milks than those in Le+Se- milks. ${ }^{34,44}$ The difference in correlations between $\mathrm{LN}(\mathrm{n}) \mathrm{T}$ or $\mathrm{LN}(\mathrm{n}) \mathrm{H}$ with milk group 1 and milk group 2 implied that the $S e$ gene is only one of the 
multiple factors influencing the actual concentrations of these neutral core HMOs in mother milk. The negative correlations between ( $\alpha 1-2)$ - and ( $\alpha 1-3 / 4)$-fucosylated HMOs in milk group 1 samples were in line with donor- and acceptorsubstrate competition between $\alpha 1,-2$-fucosyltransferase (FUT2) and $\alpha 1,-3 / 4$-fucosyltransferase (FUT3), as suggested by Samuel et al. ${ }^{42}$ It can be seen from our results that FUT2 had higher activity in early lactation, and FUT3 caught up at a later stage, which was reflected by the declining concentrations of $2^{\prime} \mathrm{FL}$ and LNFP I, while 3FL and LNFP III increased, confirming earlier literature. ${ }^{25,42,45}$ The concentration of LFNP II in milk group 1 remained constant over lactation, which could be a balance between stronger FUT3 activity and an overall decreasing synthesis of HMOs in the mammary gland. Two HMOs that contain ( $\alpha 1-3)$-linked fucose moieties at the reducing-end glucose of base structure LNT, namely, LNFP V and LNDFH II, kept constant during lactation in both milk groups, consolidating previous suggestions that their fucosylation was independent of FUT3 activity. ${ }^{42}$ The levels of $3^{\prime}$ SL, $6^{\prime}$ SL, LST, and LST b were not different between milk groups, except for a higher concentration of LST c in Secretor milk. Competition for core structures might exist between sialylation and fucosylation, as found by Samuel et al., ${ }^{42}$ although they concluded that sialylation of LNnT was less influenced by FUT activities than LNT. ${ }^{42}$ Nevertheless, the independence of lactose-derived sialylated structures ( $3^{\prime} \mathrm{SL}$ and $6^{\prime} \mathrm{SL}$ ) from Secretor status was confirmed, as reported in a previous study. ${ }^{46}$ As to the temporal changes of $3^{\prime} \mathrm{SL}$ concentrations in milk, our study showed stable concentrations in the first 3 months of life, in line with Sprenger et al. ${ }^{46}$ Note, however, that $\mathrm{Ma}$ et al. found a trend for an increase of $3^{\prime} \mathrm{SL}$ in Chinese and Malaysian populations from 2 months to 1 year of age. ${ }^{45}$ Furthermore, concentrations of $6^{\prime}$ SL, LST c, and LST b, which belonged to the $(\alpha 2-6)$-sialylated HMO group, all declined, albeit their predominance over $(\alpha 2-3)$-sialylation at 2 weeks postpartum, in agreement with Austin et al. ${ }^{25}$

Longitudinal Changes of HMO Metabolization. HMOs excreted intact in infant feces were those that escaped from gut microbiota consumption or, to a smaller extent, that escaped from being absorbed; therefore, by studying the longitudinal changes of fecal HMO profiles, we could deduce the metabolic fates of different HMOs in the GI tract, as well as the development of the gut microbiota of the infants. Previously, we showed that neutral core structures had the highest degree of degradation, while fucosylated HMOs had the lowest, at around 1 month postpartum. ${ }^{27}$ The same microbial preferences were confirmed in the current study and, thanks to the longitudinal design, even found to reach up to 12 weeks postpartum. Despite the generally higher frequency of $2^{\prime} \mathrm{FL}$ and LNFP I showing lower relative abundances in infant feces compared to those in the paired human milk (Figure 4), no differences were found between degradation of $(\alpha 1-2)$ - and ( $\alpha 1-3 / 4)$-fucosylated HMOs at any time point. Di-fucosylated HMOs were more difficult to be degraded than monofucosylated ones, resulting in higher fecal levels of the former. HMOs containing ( $\alpha 2-6)$-sialic acid were more abundant than those containing ( $\alpha 2-3)$-sialic acid in milk samples, especially at early stages; however, gut microbes seemed to favor the latter structural group as fermentation substrates, with their assimilation abilities even stronger with time. Ingested HMOs were mostly catabolized by Bifidobacterium, the most predominant bacteria in early life, with varying abilities to utilize HMOs among species and strains. ${ }^{15}$ Already in in vitro experiments, consumptions of HMOs by Bifidobacteria and Bacteroides were found to be structure-specific. ${ }^{30}$ The same observations have been reported by in vivo studies. LNT and LNnT, for instance, were two of the most consumed HMOs as indicated by their lower level or even absence in feces, ${ }^{25,47}$ similarly to the ( $\alpha 1-2)$-fucosylated HMO.$^{26}$ De Leoz et al. also found a lower microbial degradation level of LNFP II, compared to LNFP I/III/V, in the first 13 weeks of age. ${ }^{26}$

Next to a general trend of consumption of HMO structural groups, each infant displayed variation in their development trajectory of consumption patterns from two weeks to 12 weeks. We recognized three consumption patterns among 1month-old babies in a previous study, ${ }^{27}$ including complete consumption, specific assimilation of core and sialylated HMOs, or no specific degradation with all HMOs still present at considerable levels. Taking the three time points of the present study into consideration, almost all infants showed growing abilities to degrade HMOs with increased duration of breast-feeding, with several infants having already developed the gut microbiota to completely utilize all ingested HMOs at 2 or 6 weeks postpartum. This transition reflected the development of infant gut microbiota from a non-HMOconsuming population, acquired from the mothers or surrounding environment after delivery, toward a population dominated by HMO-consuming microbes. ${ }^{26}$ The infant fecal microbiota compositions of the same set of BINGO samples were analyzed by Borewicz et al., ${ }^{33}$ and a colonization pattern toward Bifidobacterium-dominated microbial communities in these infant fecal samples was found. By examining the HMO metabolization in the current study, we found that the speed of this above-mentioned transition across developmental stages was individual-dependent, since almost half of the infants had not reached the final stage yet at the last time point, 12 weeks postpartum. This interindividual variation in developmental trajectories was also noticed in other studies. ${ }^{14,28,30}$ The current study found delivery mode to be one factor that influenced the transition speed, with infants delivered by caesarean section displaying delayed colonization and enrichment of HMO-consuming microbes compared to those vaginally delivered, in consistence with the literature. ${ }^{12,19}$ Besides delivery mode, infants who were delivered at home tended to show a faster transition to reach the final stage, compared to those exposed to hospital or clinic environment at birth. The influence of delivery mode on infant gut microbiota was also proved by Borewicz et al. based on fecal microbiota of these same "BINGO" infants; however, no significant difference between different delivery places were found there. ${ }^{33}$ Nevertheless, all of these findings indicate the importance of initial colonization of infant gut for the metabolization of HMOs. Our results did not find any obvious differences in HMO metabolization transition between infants fed with Secretor or non-Secretor milk, although some studies indicated a negative impact of feeding non-Secretor milk on the gut development, ${ }^{16}$ especially those delivered by caesarean section. ${ }^{43}$ Furthermore, our results did not find significant differences in HMO metabolization between breast-fed and mix-fed infants, although feeding infants with infant formula to replace human milk is believed to significantly alter infant gut microbiota. ${ }^{18,48,49}$ This could be partly due to the exclusion of entirely formula-fed subjects, and the limited number of mixedfeeding subjects. Prebiotics, such as galacto- and fructooligosaccharides, supplemented to infant formula nowadays, 
might partially alleviate the negative influence on gut microbial maturation due to lack of HMOs. ${ }^{13}$

A limitation of the current study is the lack of Lewis-negative mothers and their babies, which could have provided more insights of enzymatic regulation of HMO synthesis as influenced by FUT3 activity. More subjects from milk group 2 with more variations regarding delivery mode and delivery place should be included in future studies, to draw solid conclusions about how these variables possibly affect infant gut microbiota and fecal HMO profiles. Furthermore, data about infants' body growth and development, as well as health status, should be followed, to find any biological significance of these differences in HMO consumption development.

To conclude, with more participants and samples from multiple time points, we were able to follow the temporal changes of HMO synthesis in mother milk, as well as HMO metabolization by paired infant gut microbiota, during the first 3 months of life. Lactation duration had a significant influence on concentrations of individual HMOs in mother milk, as well as mothers' Lewis/Secretor status, especially on the fucosylated and neutral core HMOs, and LST c. The diverse HMO metabolization patterns by the infant gut microbiota found in the current study further confirmed the observations of our previous pilot study and indicated the different stages of gut microbiota development. The transition of HMO metabolization from a low/nonspecific pattern toward a complete utilization pattern is individual-dependent and can be partly attributed to differences in mode and place of delivery. Although it remains unknown how the gut microbiota developmental pattern and HMO metabolization stages would link to long-term health outcomes, more attention is suggested to be paid on personalized nutrition, to provide infants with the most appropriate HMOs at specific developmental stage. Furthermore, HMOs with different structures were utilized with different preferences by infant gut microbiota, which could lead researchers and product developers to identify specific compounds of interest to be applied into infant nutrition, with right dosing at a given stage of growth.

\section{ASSOCIATED CONTENT}

\section{SI Supporting Information}

The Supporting Information is available free of charge at https://pubs.acs.org/doi/10.1021/acs.jafc.0c07484.

Concentrations of different HMO structural groups in infant fecal samples, from milk group 1 or milk group 2, at three time points (Figure S1); relative abundances of 3 -fucosyllactose (3FL), $2^{\prime}$-fucosyllactose $\left(2^{\prime} \mathrm{FL}\right)$, or sum of $3-\mathrm{FL}$ and $2^{\prime} \mathrm{FL}$ in mother milk and infant fecal samples, at three time points (Figure S2) (PDF)

\section{AUTHOR INFORMATION}

\section{Corresponding Author}

Henk A. Schols - Laboratory of Food Chemistry, Wageningen University, 6700 AA Wageningen, The Netherlands; (1) orcid.org/0000-0002-5712-1554; Phone: +31

317482239; Email: henk.schols@wur.nl

\footnotetext{
Authors

Fangjie Gu - Laboratory of Food Chemistry, Wageningen University, 6700 AA Wageningen, The Netherlands
}

Shuang Wang - Laboratory of Food Chemistry, Wageningen University, 6700 AA Wageningen, The Netherlands

Roseriet Beijers - Department of Developmental Psychology, Behavioral Science Institute, Radboud University, $6500 \mathrm{HE}$ Nijmegen, The Netherlands; Department of Cognitive Neuroscience, Donders Institute for Brain, Cognition and Behavior, Radboud University Medical Center, 6500 GL Nijmegen, The Netherlands

Carolina de Weerth - Department of Cognitive Neuroscience, Donders Institute for Brain, Cognition and Behavior, Radboud University Medical Center, 6500 GL Nijmegen, The Netherlands

Complete contact information is available at:

https://pubs.acs.org/10.1021/acs.jafc.0c07484

\section{Funding}

This project is jointly financed by the Benefit for the Topconsortia for Knowledge \& Innovation Agri\&Food of The Ministry of Economic Affairs, Avebe U.A., FrieslandCampina B.V., Trouw Nutrition, Nutricia Research B.V., Sensus B.V., and Winclove B.V., as coordinated by the Carbohydrate Competence Centre. C.d.W.'s work was supported by the Netherlands Organization for Scientific Research VICI (016.Vici. 185.038) grant, and a Jacobs Foundation Advanced Research Fellowship. R.B. was supported by a Netherlands Organization for Scientific Research VENI grant (016.195.197) and a Sara van Dam Project Grant of the Royal Netherlands Academy of Arts and Sciences.

\section{Notes}

The authors declare no competing financial interest.

\section{ACKNOWLEDGMENTS}

The authors acknowledge the families who generously gave their time to participate in the BINGO study. They also thank Zhibin Liu from Wageningen University for his assistance with the statistical analysis.

\section{ABBREVIATIONS}

2'FL, 2'-fucosyllactose; 3FL, 3-fucosyllactose; 3'SL, 3'sialyllactose; 6' $\mathrm{SL}, 6^{\prime}$-sialyllactose; $\mathrm{ACN}$, acetonitrile; BINGO, Biological Influences on Baby's Health and Development (in Dutch); DFL, difucosyllactose; Fuc, fucose; FUT2, $\alpha 1$,-2-fucosyltransferase; FUT3, $\alpha 1$,-3/4-fucosyltransferase; GI, gastrointestinal; HCA, hierarchical cluster analysis; HMOs, human milk oligosaccharides; HPAEC-PAD, high-performance anion exchange chromatography-pulsed amperometric detection; Le/Se, Lewis/Secretor; LNDFH, lacto- $N$-difucohexaose; LNFP, lacto- $N$-fucopentaose; $\mathrm{LNH}$, lacto- $\mathrm{N}$-hexaose; $\mathrm{LNnH}$, lacto- $N$-neohexaose; LNT, lacto- $N$-tetraose; LST, sialyl-lacto$N$-tetraose; MRPP, multiple response permutation procedures; $\mathrm{m} / z$, mass-to-charge ratios; Neu5Ac, $N$-acetylneuraminic acid; PGC-LC-MS, porous graphitized carbon-liquid chromatography-mass spectrometry; SD, standard deviation; SPE, solidphase extraction

\section{REFERENCES}

(1) Bode, L. Human milk oligosaccharides: every baby needs a sugar mama. Glycobiology 2012, 22, 1147-1162.

(2) Kulinich, A.; Liu, L. Human milk oligosaccharides: The role in the fine-tuning of innate immune responses. Carbohydr. Res. 2016, $432,62-70$. 
(3) Jost, T.; Lacroix, C.; Braegger, C.; Chassard, C. Impact of human milk bacteria and oligosaccharides on neonatal gut microbiota establishment and gut health. Nutr. Rev. 2015, 73, 426-437.

(4) Berger, P. K.; Plows, J. F.; Jones, R. B.; Alderete, T. L.; Yonemitsu, C.; Poulsen, M.; Ryoo, J. H.; Peterson, B. S.; Bode, L.; Goran, M. I. Human milk oligosaccharide 2'-fucosyllactose links feedings at 1 month to cognitive development at 24 months in infants of normal and overweight mothers. PLoS One 2020, 15, No. e0228323.

(5) Wang, B. Molecular mechanism underlying sialic acid as an essential nutrient for brain development and cognition. Adv. Nutr. 2012, 3, 465s-472s.

(6) Gnoth, M. J.; Kunz, C.; Kinne-Saffran, E.; Rudloff, S. Human milk oligosaccharides are minimally digested in vitro. J. Nutr. 2000, 130, 3014-3020.

(7) Engfer, M. B.; Stahl, B.; Finke, B.; Sawatzki, G.; Daniel, H. Human milk oligosaccharides are resistant to enzymatic hydrolysis in the upper gastrointestinal tract. Am. J. Clin. Nutr. 2000, 71, 15891596.

(8) Rudloff, S.; Pohlentz, G.; Borsch, C.; Lentze, M. J.; Kunz, C. Urinary excretion of in vivo (1)(3)C-labelled milk oligosaccharides in breastfed infants. Br. J. Nutr. 2012, 107, 957-963.

(9) Dotz, V.; Rudloff, S.; Blank, D.; Lochnit, G.; Geyer, R.; Kunz, C. ${ }^{13} \mathrm{C}$-labeled oligosaccharides in breastfed infants' urine: individual-, structure- and time-dependent differences in the excretion. Glycobiology 2014, 24, 185-194.

(10) De Leoz, M. L.; Wu, S.; Strum, J. S.; Ninonuevo, M. R.; Gaerlan, S. C.; Mirmiran, M.; German, J. B.; Mills, D. A.; Lebrilla, C. B.; Underwood, M. A. A quantitative and comprehensive method to analyze human milk oligosaccharide structures in the urine and feces of infants. Anal. Bioanal. Chem. 2013, 405, 4089-4105.

(11) Albrecht, S.; Schols, H. A.; van den Heuvel, E. G.; Voragen, A. G.; Gruppen, H. CE-LIF-MS n profiling of oligosaccharides in human milk and feces of breast-fed babies. Electrophoresis 2010, 31, 12641273.

(12) Shao, Y.; Forster, S. C.; Tsaliki, E.; Vervier, K.; Strang, A.; Simpson, N.; Kumar, N.; Stares, M. D.; Rodger, A.; Brocklehurst, P.; Field, N.; Lawley, T. D. Stunted microbiota and opportunistic pathogen colonization in caesarean-section birth. Nature 2019, 574, $117-121$.

(13) Borewicz, K.; Suarez-Diez, M.; Hechler, C.; Beijers, R.; de Weerth, C.; Arts, I.; Penders, J.; Thijs, C.; Nauta, A.; Lindner, C.; Van Leusen, E.; Vaughan, E. E.; Smidt, H. The effect of prebiotic fortified infant formulas on microbiota composition and dynamics in early life. Sci. Rep. 2019, 9, No. 2434.

(14) Chong, C. Y. L.; Bloomfield, F. H.; O'Sullivan, J. M. Factors Affecting Gastrointestinal Microbiome Development in Neonates. Nutrients 2018, 10, No. 274.

(15) Sakanaka, M.; Gotoh, A.; Yoshida, K.; Odamaki, T.; Koguchi, H.; Xiao, J. Z.; Kitaoka, M.; Katayama, T. Varied Pathways of Infant Gut-Associated Bifidobacterium to Assimilate Human Milk Oligosaccharides: Prevalence of the Gene Set and Its Correlation with Bifidobacteria-Rich Microbiota Formation. Nutrients 2020, 12, No. 71.

(16) Lewis, Z. T.; Totten, S. M.; Smilowitz, J. T.; Popovic, M.; Parker, E.; Lemay, D. G.; Van Tassell, M. L.; Miller, M. J.; Jin, Y. S.; German, J. B.; Lebrilla, C. B.; Mills, D. A. Maternal fucosyltransferase 2 status affects the gut bifidobacterial communities of breastfed infants. Microbiome 2015, 3, No. 13.

(17) Castanys-Muñoz, E.; Martin, M. J.; Vazquez, E. Building a Beneficial Microbiome from Birth. Adv. Nutr. 2016, 7, 323-330.

(18) Moore, R. E.; Townsend, S. D. Temporal development of the infant gut microbiome. Open Biol. 2019, 9, No. 190128.

(19) Cooper, P.; Bolton, K. D.; Velaphi, S.; de Groot, N.; EmadyAzar, S.; Pecquet, S.; Steenhout, P. Early Benefits of a Starter Formula Enriched in Prebiotics and Probiotics on the Gut Microbiota of Healthy Infants Born to HIV+ Mothers: A Randomized Double-Blind Controlled Trial. Clin. Med. Insights: Pediatr. 2016, 10, 119-130.
(20) Kobata, A. Structures and application of oligosaccharides in human milk. Proc. Jpn. Acad., Ser. B 2010, 86, 731-747.

(21) Wu, S.; Grimm, R.; German, J. B.; Lebrilla, C. B. Annotation and structural analysis of sialylated human milk oligosaccharides. $J$. Proteome Res. 2011, 10, 856-868.

(22) Wu, S.; Tao, N.; German, J. B.; Grimm, R.; Lebrilla, C. B. Development of an annotated library of neutral human milk oligosaccharides. J. Proteome Res. 2010, 9, 4138-4151.

(23) Urashima, T.; Hirabayashi, J.; Sato, S.; Kobata, A. Human Milk Oligosaccharides as Essential Tools for Basic and Application Studies on Galectins. Trends Glycosci. Glycotechnol. 2018, 30, SE51-SE65.

(24) Albrecht, S.; Schols, H. A.; van Zoeren, D.; van Lingen, R. A.; Groot Jebbink, L. J. M.; van den Heuvel, E. G. H. M.; Voragen, A. G. J.; Gruppen, H. Oligosaccharides in feces of breast- and formula-fed babies. Carbohydr. Res. 2011, 346, 2173-2181.

(25) Austin, S.; De Castro, C. A.; Benet, T.; Hou, Y.; Sun, H.; Thakkar, S. K.; Vinyes-Pares, G.; Zhang, Y.; Wang, P. Temporal Change of the Content of 10 Oligosaccharides in the Milk of Chinese Urban Mothers. Nutrients 2016, 8, No. 346.

(26) De Leoz, M. L. A.; Kalanetra, K. M.; Bokulich, N. A.; Strum, J. S.; Underwood, M. A.; German, J. B.; Mills, D. A.; Lebrilla, C. B. Human milk glycomics and gut microbial genomics in infant feces show a correlation between human milk oligosaccharides and gut microbiota: a proof-of-concept study. J. Proteome Res. 2015, 14, 491502.

(27) Gu, F.; Ten Kate, G. A.; Lindner, C.; Arts, I.; Penders, J.; Thijs, C.; van Leeuwen, S. S.; Schols, H. A. Combining HPAEC-PAD, PGCLC-MS and $1 \mathrm{D}{ }^{1} \mathrm{H}$ NMR to Investigate Metabolic Fates of Human Milk Oligosaccharides in One-Month Old Infants: A Pilot Study. J. Agric. Food Chem. Accepted, 2021.

(28) Albrecht, S.; Schols, H. A.; van den Heuvel, E. G.; Voragen, A. G.; Gruppen, H. Occurrence of oligosaccharides in feces of breast-fed babies in their first six months of life and the corresponding breast milk. Carbohydr. Res. 2011, 346, 2540-2550.

(29) Chaturvedi, P.; Warren, C. D.; Buescher, C. R.; Pickering, L. K.; Newburg, D. S. Survival of human milk oligosaccharides in the intestine of infants. Adv. Exp. Med. Biol. 2001, 501, 315-323.

(30) Dotz, V.; Adam, R.; Lochnit, G.; Schroten, H.; Kunz, C. Neutral oligosaccharides in feces of breastfed and formula-fed infants at different ages. Glycobiology 2016, 26, 1308-1316.

(31) Hechler, C.; Borewicz, K.; Beijers, R.; Saccenti, E.; RiksenWalraven, M.; Smidt, H.; De Weerth, C. Association between Psychosocial Stress and Fecal Microbiota in Pregnant Women. Sci. Rep. 2019, 9, No. 4463.

(32) Hechler, C.; Beijers, R.; Riksen-Walraven, J. M.; De Weerth, C. Are cortisol concentrations in human breast milk associated with infant crying? Dev. Psychobiol. 2018, 60, 639-650.

(33) Borewicz, K.; Gu, F.; Saccenti, E.; Hechler, C.; Beijers, R.; de Weerth, C.; van Leeuwen, S. S.; Schols, H. A.; Smidt, H. The association between breastmilk oligosaccharides and faecal microbiota in healthy breastfed infants at two, six, and twelve weeks of age. Sci. Rep. 2020, 10, No. 4270.

(34) Azad, M. B.; Robertson, B.; Atakora, F.; Becker, A. B.; Subbarao, P.; Moraes, T. J.; Mandhane, P. J.; Turvey, S. E.; Lefebvre, D. L.; Sears, M. R.; Bode, L. Human Milk Oligosaccharide Concentrations Are Associated with Multiple Fixed and Modifiable Maternal Characteristics, Environmental Factors, and Feeding Practices. J. Nutr. 2018, 148, 1733-1742.

(35) Elwakiel, M.; Hageman, J. A.; Wang, W.; Szeto, I. M.; van Goudoever, J. B.; Hettinga, K. A.; Schols, H. A. Human Milk Oligosaccharides in Colostrum and Mature Milk of Chinese Mothers: Lewis Positive Secretor Subgroups. J. Agric. Food Chem. 2018, 66, 7036-7043.

(36) Thurl, S.; Henker, J.; Siegel, M.; Tovar, K.; Sawatzki, G. Detection of four human milk groups with respect to Lewis blood group dependent oligosaccharides. Glycoconjugate J. 1997, 14, 795799.

(37) van Leeuwen, S. S.; Schoemaker, R. J.; Gerwig, G. J.; van Leusen-van Kan, E. J.; Dijkhuizen, L.; Kamerling, J. P. Rapid milk 
group classification by $1 \mathrm{H}$ NMR analysis of Le and $\mathrm{H}$ epitopes in human milk oligosaccharide donor samples. Glycobiology 2014, 24, $728-739$.

(38) Thurl, S.; Munzert, M.; Henker, J.; Boehm, G.; Muller-Werner, B.; Jelinek, J.; Stahl, B. Variation of human milk oligosaccharides in relation to milk groups and lactational periods. Br. J. Nutr. 2010, 104, 1261-1271.

(39) Thurl, S.; Munzert, M.; Boehm, G.; Matthews, C.; Stahl, B. Systematic review of the concentrations of oligosaccharides in human milk. Nutr. Rev. 2017, 75, 920-933.

(40) Nijman, R. M.; Liu, Y.; Bunyatratchata, A.; Smilowitz, J. T.; Stahl, B.; Barile, D. Characterization and Quantification of Oligosaccharides in Human Milk and Infant Formula. J. Agric. Food Chem. 2018, 66, 6851-6859.

(41) Hong, Q.; Ruhaak, L. R.; Totten, S. M.; Smilowitz, J. T.; German, J. B.; Lebrilla, C. B. Label-free absolute quantitation of oligosaccharides using multiple reaction monitoring. Anal. Chem. 2014, 86, 2640-2647.

(42) Samuel, T. M.; Binia, A.; de Castro, C. A.; Thakkar, S. K.; Billeaud, C.; Agosti, M.; Al-Jashi, I.; Costeira, M. J.; Marchini, G.; Martinez-Costa, C.; Picaud, J. C.; Stiris, T.; Stoicescu, S. M.; Vanpee, M.; Domellof, M.; Austin, S.; Sprenger, N. Impact of maternal characteristics on human milk oligosaccharide composition over the first 4 months of lactation in a cohort of healthy European mothers. Sci. Rep. 2019, 9, No. 11767.

(43) Korpela, K.; Salonen, A.; Hickman, B.; Kunz, C.; Sprenger, N.; Kukkonen, K.; Savilahti, E.; Kuitunen, M.; de Vos, W. M. Fucosylated oligosaccharides in mother's milk alleviate the effects of caesarean birth on infant gut microbiota. Sci. Rep. 2018, 8, No. 13757.

(44) Tonon, K. M.; de Morais, M. B.; Abrao, A. C. F. V.; Miranda, A.; Morais, T. B. Maternal and Infant Factors Associated with Human Milk Oligosaccharides Concentrations According to Secretor and Lewis Phenotypes. Nutrients 2019, 11, No. 1358.

(45) Ma, L.; McJarrow, P.; Jan Mohamed, H. J. B.; Liu, X.; Welman, A.; Fong, B. Y. Lactational changes in the human milk oligosaccharide concentration in Chinese and Malaysian mothers' milk. Int. Dairy J. 2018, 87, 1-10.

(46) Sprenger, N.; Lee, L. Y.; De Castro, C. A.; Steenhout, P.; Thakkar, S. K. Longitudinal change of selected human milk oligosaccharides and association to infants' growth, an observatory, single center, longitudinal cohort study. PLoS One 2017, 12, No. e0171814.

(47) Davis, J. C.; Totten, S. M.; Huang, J. O.; Nagshbandi, S.; Kirmiz, N.; Garrido, D. A.; Lewis, Z. T.; Wu, L. D.; Smilowitz, J. T.; German, J. B.; Mills, D. A.; Lebrilla, C. B. Identification of Oligosaccharides in Feces of Breast-fed Infants and Their Correlation with the Gut Microbial Community. Mol. Cell. Proteomics 2016, 15, 2987-3002.

(48) Davis, E. C.; Dinsmoor, A. M.; Wang, M.; Donovan, S. M. Microbiome Composition in Pediatric Populations from Birth to Adolescence: Impact of Diet and Prebiotic and Probiotic Interventions. Dig. Dis. Sci. 2020, 65, 706-722.

(49) Verkhnyatskaya, S.; Ferrari, M.; de Vos, P.; Walvoort, M. T. C. Shaping the Infant Microbiome With Non-digestible Carbohydrates. Front. Microbiol. 2019, 10, No. 343. 